\title{
A New Journal Helping to Shape the Future of Materials
}

\author{
Huseyin Sehitoglu
}

Published online: 6 March 2015

(c) ASM International 2015

Shape memory phenomenon refers to the exceptional quality of some materials which exhibit the ability to reverse deformation upon heating and cooling after inelastic deformation. Since most deformations beyond the yield strain are irrecoverable in materials, shape memory alloys' characteristics are rather unusual. They have been exploited in many applications ranging from biomedical (stents) to automotive (actuators and sensing). The shape memory field has remained vibrant in several fronts in the last six decades starting with the work on $\mathrm{Au}-\mathrm{Cd}$ - and $\mathrm{Cu}$-based alloys in the 1950s and subsequently the discovery of NiTi in the early 1960s. The discovery of the shape memory phenomenon in Fe-based alloys followed in the 1970s. More recently in the 1990s and 2000s, increasing emphasis on ferromagnetic shape memory (the NiMnGa and $\mathrm{NiFeGa}$ type), iron-based shape memory alloys (FeNiCoX variety), high-temperature shape memory alloys (NiTiHf/Pt/Pd), and potential biomedical alloys (TiNb type and others) followed. In addition, there are examples of polymers and ceramics exhibiting unusual shape memory characteristics as well.

The field has been undergoing a renaissance in the last 10 years, which is evident through increased publication rates (more than 3,000 articles per year) and multiple symposia (exceeding ten) per year. This is partly because of intense intellectual curiosity surrounding these alloys exhibiting such unusual properties, the availability of more powerful experimental and computer tools, and the commercial

Huseyin Sehitoglu, Editor-in-Chief-Nyquist Chair, University of Illinois.

H. Sehitoglu $(\square)$

Department of Mechanical Science and Engineering, University of Illinois, Urbana, IL 61801, USA

e-mail: huseyin@illinois.edu

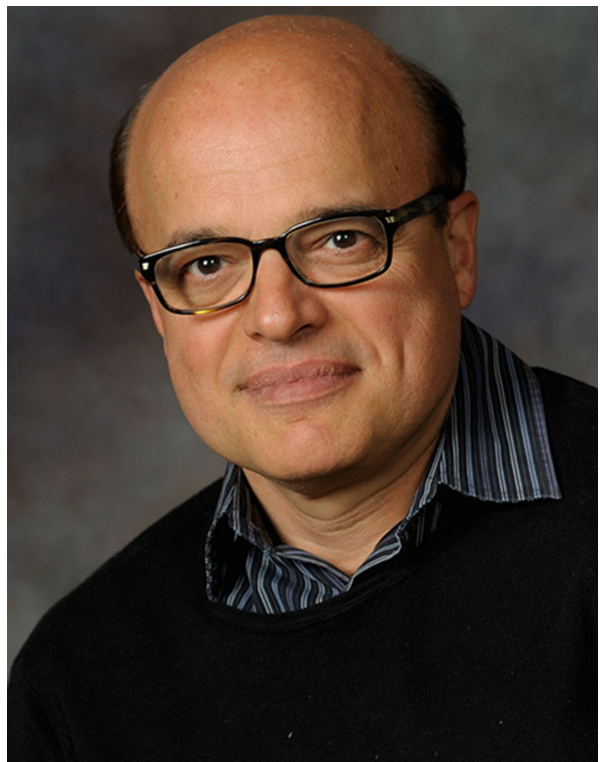

importance of these alloys in the biomedical, aerospace, and other industries. In addition to the materials science and mechanics communities, the applied mathematics and applied physics communities have contributed to a sound fundamental knowledge in the field. Shape Memory and Superelasticity will therefore provide a natural home for papers from authors with diverse disciplinary backgrounds in engineering and sciences addressing shape memory phenomena.

To elaborate further, new materials with exceptional properties have been discovered recently, adding to the repertoire of the well-known shape memory alloys. Researchers of these new materials have put out quality studies keeping the field advancing. These works emphasized both functional and mechanical properties of shape 
memory materials. The functional properties represent the superelastic and shape memory response under mechanical, magnetic, and temperature fields exploited for damping, actuation, and clamping applications. The chemical compositions, heat treatments, and processing routes dictate the transformation strains, the transformation temperatures, and stress/temperature hysteresis in shape memory metallic alloys. The mechanical properties refer to the intrinsic stresses at the onset of plasticity in austenitic and martensitic domains and their dependence on temperature, the magnitude of ductility, the moduli of austenite and martensites, the fatigue crack growth behavior such as threshold levels and fracture stress, and so on. The functional and mechanical properties are intimately linked at the microstructural level upon formation of martensite variants on specific planes, their interaction with slip, the internal stresses, and residual strains that result. In addition, there are grain boundary effects, and the role of secondary phases and precipitates on both functional and mechanical properties that can be rather complex.

To develop a better understanding of shape memory phenomena, specialized experimental and theoretical tools have been utilized recently to glean further insight. The experimental tools include high-resolution strain measurements such as digital image correlation and high energy diffraction methods to identify the evolution of phases and twin types. Theoretical tools, such as atomistic calculations, provided unprecedented insight into transition states unraveling the complex shears and shuffles occurring during transformation. The differences in energies between the austenite and martensite phases are rather small, allowing transformation at stresses of the order of MPas rather than GPas. At the macroscale, continuum mechanics tools informed with atomistics resulted in the improvement of mechanical response predictions. Further improvements are necessary to properly account for tension-compression asymmetry and crystal orientation effects. Such a confluence of resources ranging from atomistics to macroscale testing and modeling is expected to advance the shape memory field in the future and characterize potential materials rapidly. Therefore, this journal aims to provide the forum for communication and education among experts and students from diverse backgrounds to address phenomena spanning a multitude of length scales.

Shape memory alloys have a huge potential, but at the same time, there is a strong need for research for realization of this potential. We touch on some of the exciting developments and technical challenges below. The utilization of shape memory alloys in biomedical applications calls for better understanding of fatigue and fracture properties and processing routes to achieve desired textures and surfaces. Conventional fatigue and fracture methodologies need revision when working with shape memory alloys. Recently, the possibility of utilizing the shape memory alloys for adiabatic cooling (elastocaloric effects) during reverse transformation has presented new opportunities. The development of higher entropy change SMAs is needed to realize higher temperature changes. There has also been a strong emphasis on high-temperature shape memory alloys for aerospace applications with the addition of ternary elements.

The challenges are to develop more ductile alloys that can be used in high temperature tension applications and also to lower cost. The iron-based shape memory alloys with potentially lower cost applications in civil engineering are of interest. Traditionally, the iron-based shape memory alloys have had very wide hysteresis, and superelasticity was difficult to realize. In the last few years, there have been several compositions that exhibit superb superelasticity at room temperature. The titanium-based alloys are excellent alternatives to current shape memory alloys as they permit increased biocompatibility. The challenges lie in increasing the transformation strains and improving the ductility via heat treatment and compositional changes in these materials. The ferromagnetic shape memory alloys with potential rapid actuation under magnetic fields also have been of considerable interest to the community as they provide a faster response time compared to conventional shape memory alloys. The increase in ductility of such alloys can open new possibilities for applications. The potential for shape memory phenomenon in nonmetals, including polymers and ceramics, nanograined materials, and materials with special grain boundaries and unusual shape memory properties have also recently been recognized. The discussion and challenges above provide only a glimpse of the enormous potential of these classes of metallic and non-metallic materials. By establishing the journal Shape Memory and Superelasticity, we plan to draw papers from diverse communities to improve our understanding of shape memory alloys and to facilitate utilization of shape memory alloys in applications.

Currently, the work on shape memory materials spans across a wide range of journals. The idea of establishing a journal with an exclusive focus on shape memory alloys was received well by the community, as evidenced by responses to surveys conducted by ASM during the past 18 months. Both industrial and academic researchers desire to see a journal containing content on fundamental developments, innovative manufacturing, and novel use of these materials. Shape Memory and Superelasticity is being launched with two special inaugural issues in 2015 containing more than 30 invited papers authored by experts in the field. The journal is being published under the aegis of ASM International and the International Organization on Shape Memory and Superelastic Technologies (SMST). It will publish papers of high quality and will consider works from all disciplines of science and engineering related to 
shape memory. The journal will publish works that advance new concepts, new experiments, and new theories with a scholarly approach. Many conferences are being organized every year that include emerging topics, such as high-temperature shape memory and ferromagnetic shape memory alloys. These are in addition to the conferences on martensitic transformations. It is worth noting that shape memory presentations constitute a large fraction of "martensitic transformation" conferences because of the growing interest in this field. Shape Memory and Superelasticity will consider manuscripts based on papers that have been presented at the SMST event or other conferences that are of significant interest to the community. All papers will need to conform to the high standards of the journal.
I would like to take this opportunity to thank the ASM International staff who organized the surveys leading to the decision to launch a new journal and provided guidance on all matters leading to the publication of this inaugural issue of Shape Memory and Superelasticity. SMST and the publisher Springer strongly backed the development of the journal as well. My thanks to the Associate Editors and Editorial Advisory Board Members who have agreed to volunteer their time and to the contributors of the first two issues who worked intensely to meet the deadlines. I am grateful for a very large number of people who accepted my invitation to contribute as authors. I hope you will enjoy reading the upcoming papers in the journal. 